\title{
PRODUCCIÓN DE BIOMASA DE Artemia franciscana Kellogg 1906 UTILIZANDO DIFERENTES DIETAS
}

\section{BIOMASS PRODUCTION OF Artemia franciscana Kellogg 1906 USING DIFFERENT DIETS}

\author{
Rosario Cisneros $^{1}$ y Enrique Vinatea ${ }^{2}$
}

\begin{abstract}
Resumen
Se realizó el cultivo semiintensivo de Artemia franciscana, cepa Perú, utilizando diferentes dietas en base a microalgas e insumos alimenticios que forman parte de algunos ingredientes que se utilizan para preparar alimento formulado de peces y crustáceos, con el fin de determinar la factibilidad del uso de estos para la obtención de biomasa de adultos. El estudio se ejecutó en base a un diseño experimental completamente aleatorio con 7 tratamientos y 3 réplicas cada uno, aplicándose a los datos obtenidos un Análisis de Varianza de una vía (ANOVA) y la prueba de Tukey para establecer diferencias significativas entre los tratamientos. Los resultados del análisis próximal de las dietas, mostraron que la harina de soya presentó la cantidad de proteínas requerida por $A$. franciscana durante su crecimiento (34.33\%), igualmente con esta dieta se obtuvo la mejor tasa de crecimiento (0.19), biomasa más alta (113.33 g) y el mejor Factor de Conversión Alimenticia (1.4). Así mismo la biomasa de A. franciscana producida con harina de soya, presentó un nivel de proteínas adecuado para la maduración de reproductores de crustáceos (53.38 \%).
\end{abstract}

Palabras clave: Artemia franciscana, cepa Perú, cultivo, dietas, biomasa

\begin{abstract}
Artemia franciscana, Peru strain, was cultured under different diets-based in microalgae and nutritious inputs with the aim to determine the feasibility to produce adult Artemia biomass. A completely randomized design experiment was carried out, with seven treatments and three replications each one. One way variance analysis (ANOVA) and the Turkey test were used to analyze the data to establish significant differences among treatments. The results of biochemical analysis showed that soya bean diet fulfilled the protein content needed for a proper growth of $A$. fransiscana. This diet also showed the best growth rate (0.19), highest biomass production (113.13 g), best food conversion ratio (1.4) and a protein content suited for crustacean broodstock maturation (53.38\%).
\end{abstract}

Key words: Artemia franciscana, Perú strain, culture, diets, biomass

\section{Introducción.}

En la actualidad, Artemia spp. es el microcrustáceo más empleado como alimento vivo para larvas de muchos peces y crustáceos marinos en cultivo, desde nauplio hasta la etapa adulta, (Vinatea, 1995; Persoone et al., 1980; Monroig et al., 2006; Haga et al., 2006; Godinez et al., 2004; Bransdena et al., 2005) .

La importancia de su valor nutricional radica en que es fuente rica en nutrientes, incluyendo aminoácidos esenciales y ácidos grasos altamente insaturados de la serie w3 (HUFAs w3) como el acido eicosapentaenoico (EPA, 20:5w3) y docosahexaenoico (DHA, 22:6 w3), los que son esenciales en el desarrollo normal de los nervios y la vista en estadios iniciales de larvas de peces marinos (Robin, 1995; Suprayudi et al., 2004; Bransdena et al., 2005; Monroig et al., 2007; Zelaya et al., 2007).

En acuicultura mayormente se utiliza Artemia spp. bajo la forma de nauplio recién nacido, cuyo valor nutritivo es muy alto en cuanto a lípidos
(Torrentera \& Tacón, 1989), sin embargo el empleo de animales adultos presenta numerosas ventajas, ya que éstos contienen mayor cantidad de proteinas que los nauplios (60 \% de proteínas), lo que resulta beneficioso para el acondicionamiento reproductivo de organismos en cultivo (Versichele et al., 1991).

Actualmente ha cobrado mayor importancia el uso de adultos (Verschuere et al., 1999) y existe una creciente demanda de los laboratorios productores de postlarvas de camarones peneidos, para inducir la maduración ovárica de los reproductores (Godinez et al., 2004). La biomasa de Artemia spp. también puede ser usada como ingrediente en las dietas para larvas de peces y crustáceos y su más interesante aplicación es la completa substitución del nauplio recién eclosionado por biomasa seca congelada y micronizada en la producción de camarones pendidos en "hatchery" (Sorgeloos et al., 1986).

A medida que la acuicultura ha ido progresando en el mundo, la oferta de quistes y sus productos alternativos, como biomasa viva, congelada, 
liofilizada, flakes, pellets, etc., no han logrado cubrir la gran demanda existente en la actualidad (Sorgeloos et al., 1986; Vinatea, 1999; Seoka et.al., 2007) у una amplia variedad de alimento vivo (microalgas) e inerte (harina de trigo, arroz, soya, etc.) han sido usados con éxito para su cultivo (Stottrup \& McEvoy, 2003). Debido a que la utilización de microalgas genera un mayor costo en la producción a gran escala, se ha intentado la obtención de biomasa con alimentos alternativos menos costosos (Tizol, 1994; Godinez et al., 2004), por ello el objetivo de este trabajo fue determinar la factibilidad del uso de diferentes insumos alimenticios para la obtención de biomasa de A. franciscana, cepa Perú.

\section{Materiales y métodos.}

Tratamientos/dietas.

Fueron utilizadas 7 dietas, que se prepararon en base a harinas que forman parte de algunos ingredientes que se utilizan para preparar raciones alimenticias de peces y crustáceos (Tacon, 1987) y una constituida por microalgas. Estas fueron, Harina de soya (HS), Polvillo de arroz (PA), Harina de exoesqueleto de camarón (HC), Harina de alfalfa (HA), mezcla de $\mathrm{HS}+\mathrm{HA}+\mathrm{HP}+\mathrm{HC}+\mathrm{T}$-iso (Mix) (en una proporción de $20 \%$ cada una), la microalga Isochrysis galbana (T-iso), especialmente rica en ácidos grasos de cadena larga (Napolitano et al., 1990) y Harina de pescado (HP) como control, debido a que ésta contiene todos los aminoácidos esenciales requeridos por crustáceos (Gallardo et al.,1989).. La

Tabla 1. Análisis químico proximal (\% en peso húmedo) de insumos alimenticios utilizados en el cultivo de Artemia franciscana, cepa Virrila, Perú.

\begin{tabular}{|c|c|c|c|c|c|}
\hline$\%$ & $\begin{array}{c}\text { Polvillo } \\
\text { de } \\
\text { arroz } \\
\text { (PA) }\end{array}$ & $\begin{array}{c}\text { Harina } \\
\text { de } \\
\text { soya } \\
\text { (HS) } \\
\end{array}$ & $\begin{array}{c}\text { Harina } \\
\text { de } \\
\text { camarón } \\
(\mathrm{HC})\end{array}$ & $\begin{array}{c}\text { Harina } \\
\text { de } \\
\text { alfalfa } \\
(\mathrm{HA}) \\
\end{array}$ & $\begin{array}{c}\text { Harina } \\
\text { de } \\
\text { pescado } \\
\text { (HP) } \\
\end{array}$ \\
\hline Humedad & 15.82 & 7.78 & 11.16 & 12.24 & 9.16 \\
\hline Proteina & 13.63 & 34.33 & 38.66 & 19.86 & 55.74 \\
\hline Grasa & 7.07 & 22.60 & 2.50 & 2.17 & 8.10 \\
\hline Fibra & 8.70 & 1.33 & - & 15.03 & - \\
\hline Ceniza & .30 & 4.50 & 22.15 & 13.55 & 20.25 \\
\hline ELN & 46.48 & 29.46 & 25.53 & 37.15 & 6.75 \\
\hline
\end{tabular}

HC se obtuvo de restos de caparazón del camarón de río Cryphiops caementarius, secado y triturado.

Análisis químicos de los insumos alimenticios.

Se realizó el análisis químico proximal (\% en peso húmedo) de los insumos utilizados (tabla 1). Así mismo de la biomasa obtenida con la dieta que proporcionó mayor rendimiento y la dieta control, con fines comparativos. Estos análisis se realizaron en el Laboratorio de Evaluación Nutricional de Alimentos de la Universidad Nacional Agraria La Molina.
Diseño experimental y análisis estadístico.

Se aplicó un diseño experimental completamente aleatorio, utilizando 8 tratamientos/dietas, cada una con tres repeticiones. Las unidades experimentales fueron tanques de fibra de vidrio de 300 litros. Para el cultivo se utilizó agua de mar filtrada a $1 \mu \mathrm{y}$ esterilizada por un sistema de lámparas ultravioleta para mantener la calidad del agua y evitar que alguna variación de ésta afecte los resultados (Fraga et al., 1996). La densidad de cultivo fue 150 nauplios/ml. El cultivo se clasificó como semi-intensivo, por la densidad, así como por la alimentación y estructuras de cultivo artificiales (Xunta de Galicia, 1991). Toda variación que pudo existir entre las unidades experimentales pasó a formar parte del error experimental (Cochran \& Cox, 1997; Steel \& Torrie, 1995).

Los datos de biomasa fueron sometidos a las pruebas de normalidad de Shapiro-Wilk y de homogeneidad de varianzas de Cochran (Quinn \& Keough, 2002). Luego de estas pruebas se aplicó un análisis de varianza de una vía (ANOVA) para determinar diferencias estadísticas significativas y la prueba Tukey, para comparar los valores medios de biomasa obtenida con cada tratamiento y determinar cuales presentaban diferencias (Fraga et al., 1996; Godinez et al., 2004).

Conducción del experimento: manejo y alimentación.

Las dietas fueron suministradas ad libitum 3 veces al día (9:00, 13:00 y 17:00 h), ya que la literatura define para crustáceos una frecuencia de 2 a 3 raciones por día para minimizar la lixiviación de nutrientes en el agua (Zimmermann et al., 1989). El suministro de alimento inerte se inició con $1 \mathrm{~g}$, el que fue aumentando en promedio de 0.5 a $1 \mathrm{~g}$ con respecto al suministro del día anterior, en la tabla 2 se observa el total de alimento consumido en todos los tratamientos.

La microalga T-iso fue adicionada como complemento junto con las harinas (por su alto contenido de ácidos grasos), a una concentración inicial de $1.43 \times 10^{6} \mathrm{cel} / \mathrm{ml}$ (0.09 g). Para determinar el peso en gramos de la microalga, las células fueron retenidas en malla de 10 micras y luego se determinó el peso húmedo.

Tabla 2. Alimento consumido (g) durante el cultivo semiintensivo de Artemia franciscana cepa Virrilá, Perú, con diferentes dietas.

\begin{tabular}{ccc}
\hline Dieta & $\begin{array}{c}\text { Consumo } \\
\text { (g) } \\
\text { Microalga }\end{array}$ & Insumo \\
\hline HS & 4.38 & 159.50 \\
HC & 4.61 & 220.50 \\
T-iso & 63.12 & - \\
PA & 19.61 & 191.00 \\
HA & 18.66 & 192.00 \\
HP & 8.89 & 116.00 \\
Mix & 9.31 & 136.00 \\
\hline
\end{tabular}

Los tanques fueron mantenidos en un ambiente 
tipo vivero, con aireación constante y un régimen de iluminación permanente, en base a 10 horas de luz natural y 14 horas de luz artificial, con el fin de proporcionar luz a las microalgas para la fotosíntesis, mantener una temperatura cálida, ya que la mayoría de cepas de Artemia spp. prefieren rangos de 19 a $25{ }^{\circ} \mathrm{C}$ (Van Stappen, 1996; Dohnt \& Van Stappen, 2003), pues esta tiene un efecto significativo sobre el crecimiento y supervivencia (Lavens \& Sorgeloos, 1996), además de evitar variaciones drásticas de este parámetro durante la noche.

Se llevó un registro diario del crecimiento promedio en longitud (mm) de los ejemplares de A. franciscana sometidos a los diferentes tratamientos. Así mismo diariamente se midieron parámetros fisicoquímicos como temperatura, salinidad y pH. En forma semanal se determinó el contenido de Oxígeno disuelto (OD) y Demanda Bioquímica de Oxígeno $\left(\mathrm{DBO}_{5}\right)$, para verificar la calidad del agua de los tanques.

Tasa de crecimiento y Factor de Conversión del alimento

Se determinó la Tasa de Crecimiento Específico de la biomasa, mediante la formula propuesta por Sorgeloos et al. (1986):

$$
\mathrm{K}=\frac{\ln \text { (Biomasa final/Biomasa inicial) }}{\mathrm{T}}
$$

Donde :

$\mathrm{K}=$ Tasa de crecimiento específico de la biomasa

$\mathrm{T}=$ Tiempo de duración del cultivo

Se pesó el número total de nauplios al inicio del cultivo, con el fin de estimar la biomasa húmeda inicial promedio. La cosecha de los ejemplares se realizó a los 25 días de cultivo, al observarse un mayor porcentaje de ejemplares adultos con diferenciación sexual. El total de individuos cosechados fue pesado, estimándose la biomasa húmeda final promedio, obtenida con cada dieta. Así mismo se estimó el peso total de alimento consumido. Con estos datos se calculó el Factor de Conversión del Alimento (FCA), aplicándose la siguiente formula (Sorgeloos et al., 1986):

$$
\text { FCA }=\frac{\text { Alimento suministrado }}{\text { (Biomasa final - Biomasa inicial) }} .
$$

El experimento se realizó en el laboratorio de Cultivos Marinos del Instituto del Mar del Perú, localizado en la Provincia Constitucional del Callao, durante el periodo enero 1999 a febrero del 2000. Para la obtención de nauplios se utilizaron quistes de Artemia franciscana Kellog 1906, cepa Virrilá, Perú, los mismos que fueron producidos en estanques al aire libre por el Fondo Nacional de Desarrollo Pesquero (FONDEPES).

\section{Resultados y discusión.}

Los parámetros fisicoquímicos registrados en los tanques de cultivo se observan en la tabla 3. En
Tabla 3. Parámetros físicoquímicos (M +/-DS) registrados durante el cultivo semiintensivo de Artemia franciscana cepa Virrilá, Perú, con diferentes dietas.

\begin{tabular}{cccccc}
\hline Dieta & $\begin{array}{c}\text { Temperatura } \\
\left({ }^{\circ} \mathrm{C}\right)\end{array}$ & $\mathrm{pH}$ & $\begin{array}{c}\text { Salinidad } \\
(\text { ups })\end{array}$ & $\begin{array}{c}\text { OD } \\
(\mathrm{mg} / \mathrm{l})\end{array}$ & $\begin{array}{c}\text { DBO } 5 \\
(\mathrm{mg} / \mathrm{l})\end{array}$ \\
\hline HS & $22.35+/-0.93$ & $7.79+/-0.33$ & $36.52+/-1.11$ & $7.79+/-0.68$ & $5.25+/-0.60$ \\
HC & $22.39+/-0.92$ & $7.79+/-0.31$ & $36.40+/-1.12$ & $7.19+/-0.64$ & $5.45+/-2.56$ \\
T-iso & $22.70+/-1.63$ & $7.77+/-0.34$ & $36.61+/-1.20$ & $7.21+/-0.61$ & $3.45+/-1.74$ \\
PA & $22.70+/-1.58$ & $7.77+/-0.29$ & $36.59+/-1.18$ & $6.29+/-1.22$ & $2.58+/-0.42$ \\
HA & $22.72+/-1.65$ & $7.79+/-0.31$ & $36.63+/-1.21$ & $5.57+/-1.25$ & $3.42+/-0.74$ \\
HP & $22.44+/-0.95$ & $7.84+/-0.45$ & $36.60+/-1.08$ & $6.66+/-1.06$ & $3.71+/-1.39$ \\
Mix & $22.42+/-0.85$ & $7.74+/-0.32$ & $36.56+/-1.04$ & $8.44+/-0.25$ & $4.95+/-1.37$ \\
\hline
\end{tabular}

relación a la temperatura esta se mantuvo en valores adecuados (22.35 a $22.75{ }^{\circ} \mathrm{C}$ ) ya que Artemia spp. prefiere ambientes relativamente cálidos (Vinatea, 1999; Vinatea et al., 2002). A pesar de ser un crustáceo euritérmico, capaz de tolerar temperaturas entre 5 a $30{ }^{\circ} \mathrm{C}$ (este rango de tolerancia, depende de la cepa geográfica), cultivos en tanques también se realizan a temperaturas de 25 a $28{ }^{\circ} \mathrm{C}$ (Sorgeloos et al., 1986).

En relación al pH, valores menores a 5 y mayores a 10 son usualmente letales (Persoone et al.,1980), en este sentido Stottrup \& McEvoy (2003) manifiestan que estas toleran $\mathrm{pH}$ de 6.5 a 8, así mismo Hoff \& Snell (1993) mencionan que incluso niveles de hasta 8 y 9 son adecuados y en este trabajo los valores se mantuvieron dentro de ese margen (7.74 a 7.84).

Respecto a la salinidad, los cultivos se realizan entre 30 a 50 ups (Sorgeloos et al., 1986), sin embargo Stottrup \& McEvoy (2003) refieren que el uso de agua de mar natural (35 ups) es más práctico en los cultivos controlados. En este caso la salinidad varió de 36.40 a 36.64 ups.

En relación a los valores de OD se ha reportado que Artemia spp. puede vivir en ambientes con menos de $1 \mathrm{mg} / \mathrm{l}$ (Vinatea, 1999), sin embargo la producción de biomasa decrece a concentraciones debajo de 2 mg/l y para una optima producción son sugeridas concentraciones por encima de $2.5 \mathrm{mg} / \mathrm{l}$ (Stottrup \& McEvoy, 2003). En este caso los valores fueron altos (5.57 a $8.44 \mathrm{mg} / \mathrm{l})$, ya que la intensidad de la aireación se mantuvo mediante el uso de piedras difusoras. 
Con respecto al DBO, importante para controlar el nivel de materia orgánica, los valores fueron bajos, de 2.58 a $5.45 \mathrm{mg} / \mathrm{l}$, indicando buena calidad de agua o baja polución, lo que se consiguió mediante el suministro ad libitum del alimento. Según Stottrup \&

McEvoy (2003) lo que se recomienda como estrategia de alimentación por ser Artemia spp. un organismo filtrador-continuo, es distribuir el alimento tan frecuente como es posible para obtener un alto crecimiento, así como mínima deposición de alimento no consumido.

En la figura 1 se observa el crecimiento en longitud (mm) de A. franciscana con las diferentes dietas, observándose que con HS los ejemplares alcanzaron una mayor talla, esta fue de $5.72 \mathrm{~mm}$.

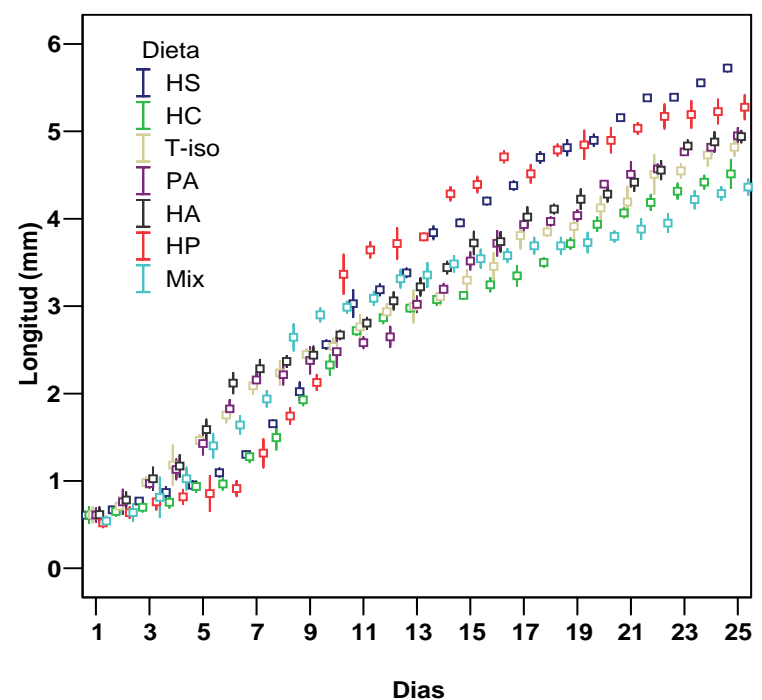

Figura 1. Crecimiento en longitud (M+/-DS) de Artemia franciscana, cepa Virrilá, Perú , durante el cultivo con diferentes dietas. Las barras indican la talla media más los intervalos de confianza al 95\%.

En relación a K, el valor más alto se obtuvo con HS (0.19) (tabla 4). En este sentido Tacón (1987 a) refiere que crustáceos en pleno crecimiento requieren $35 \%$ de proteínas, y HS presentó $34.33 \%$ en el análisis próximal (Tabla 1), lo que favoreció el rápido crecimiento de los ejemplares con esta dieta.

En la tabla 4 también se observa la biomasa obtenida con las diferentes dietas, con HS se obtuvo el mayor valor promedio (113.33 g) (figura 2). Tacon (1987 b) manifiesta que la HS es una buena fuente de aminoácidos esenciales (EAA) y una de las pocas plantas ricas en lisina, sin embargo hay que tener en cuenta que presenta proteasa, la que es un inhibidor de la tripsina y causa mortalidad en los animales, pero es totalmente desactivada a $100{ }^{\circ} \mathrm{C}$ por 15 a 20 minutos (FAO, 1983), por este motivo se recomienda suministrarla en forma cocida.

En relación al FCA, con HS se obtuvo el mayor valor promedio (1.4) (Tabla 4), similar a lo que Guitart \& González (1987) obtuvieron utilizando salvado de arroz (citado en Tizol, 1994) y a los

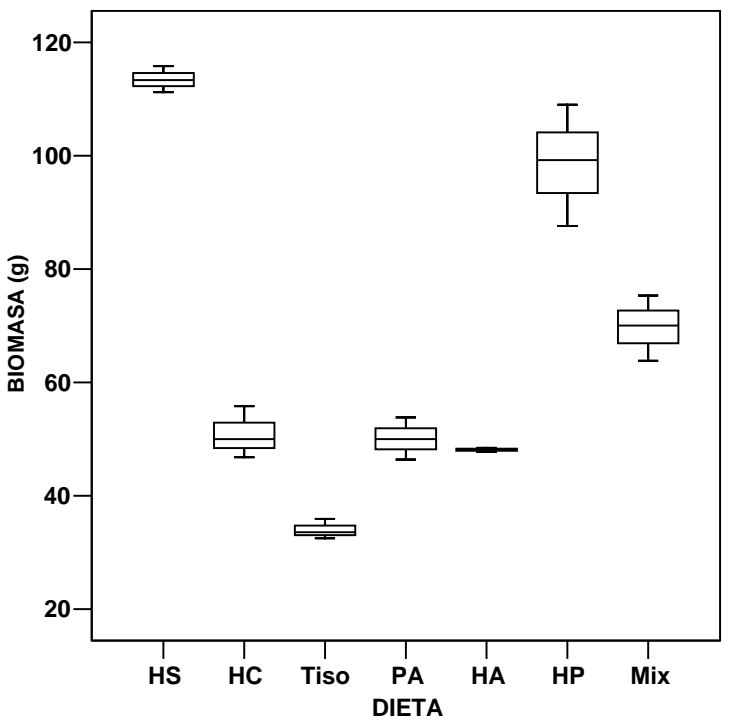

Figura 2. Biomasa obtenida durante el cultivo semiintensivo de Artemia franciscana, cepa Virrilá, Perú, durante el cultivo con diferentes dietas.

resultados de Vinatea et al. (1993), quienes obtuvieron 1.07 con harina de soya micronizada y la microalga Thalassiosira fluviatilis como alimento suplementario.

Tabla 4. Constante de crecimiento (K), Biomasa (g) y Factor de conversión del alimento (FCA) obtenidos durante el cultivo semiintensivo de Artemia franciscana cepa Virrilá, Perú con diferentes dietas.

\begin{tabular}{cccc}
\hline Dieta & K & Biomasa (g) & FCA \\
\hline HS & 0.19 & $113.33+/-2.87$ & $1.40+/-0.04$ \\
HC & 0.15 & $50.70+/-4.90$ & $4.40+/-0.43$ \\
T-iso & 0.14 & $33.57+/-1.76$ & $1.88+/-0.10$ \\
PA & 0.15 & $50.00+/-3.82$ & $4.20+/-0.33$ \\
HA & 0.15 & $48.17+/-0.64$ & $4.37+/-0.07$ \\
HP & 0.18 & $99.17+/-4.70$ & $1.28+/-0.14$ \\
Mix & 0.16 & $70.03+/-5.71$ & $2.07+/-0.17$ \\
\hline
\end{tabular}

Sin embargo difieren de lo obtenido por Tizol (1994), quien obtuvo un FCA de 2.0, utilizando levadura "torula" (derivado de la caña de azúcar).

En relación a la HC, esta presenta una fracción de proteínas solubles que desempeñan un papel desconocido, pero fundamental en la percepción del alimento (Devresse, 1999), por lo que este tipo de harina puede ser utilizada como atractante, restringiendo su uso a menos del $25 \%$ en dietas para acuicultura (FAO, 1983), debido a la presencia de quitina, la que es difícil de digerir y su contenido de nitrógeno no es utilizable. De la Higuera \& Cardenete (1987) refieren que una dieta puede tener alto contenido de proteína, pero si su digestibilidad es baja, 
no cubre las necesidades para el crecimiento. Sobre PA y HA, pese a que estos insumos se presentan en la lista de ingredientes utilizados en las dietas para cultivo de peces y crustáceos, su cantidad de fibra hace aumentar el tránsito del alimento en el sistema digestivo, no permitiendo actuar a las enzimas digestivas el tiempo suficiente (Fraga et al., 1996). En

Tabla 5. Análisis de varianza de la biomasa final obtenida en el cultivo semiintensivo de Artemia franciscana cepa Virrilá, Perú, con diferentes dietas.

\begin{tabular}{llrrrrr}
\hline & & $\begin{array}{c}\text { Suma } \\
\text { de } \\
\text { cuadrados }\end{array}$ & $\begin{array}{c}\text { Grados } \\
\text { de }\end{array}$ & $\begin{array}{c}\text { Cuadrado } \\
\text { libertad }\end{array}$ & F & Probabilidad \\
\hline $\begin{array}{l}\text { Biomasa } \\
\text { final }\end{array}$ & $\begin{array}{l}\text { Entre } \\
\text { grupos }\end{array}$ & 26139.35 & 6 & 3734.19 & 160.03 & $1.3277 \mathrm{E}-13$ \\
& $\begin{array}{l}\text { Dentro } \\
\text { de }\end{array}$ & & & & & \\
& grupos & 373.35 & 14 & 23.33 & & \\
& Total & 26512.70 & 20 & & & \\
\hline
\end{tabular}

este sentido se recomienda usar estos insumos en una dieta mixta, ya que esta proporcionó mejor biomasa y FCA, después de HS y HP.

El análisis de varianza (ANOVA) mostró diferencias significativas $(\mathrm{p}<0.05)$ entre las medias de la biomasa final obtenida con las diferentes dietas (Tabla 5).

Con la prueba de Tukey se corroboró que la biomasa obtenida con HS fue significativamente mayor. Así mismo se observó que las dietas HA, PA y HC, no presentaron diferencias significativas entre sí $(\mathrm{p}>0.05)$.

El análisis químico proximal de la biomasa obtenida con HS (Tabla 6) presentó $53.38 \%$ de proteínas, lo que resulta adecuado para reproductores de langostinos.

\begin{tabular}{|c|c|c|c|}
\hline $\begin{array}{lr}\text { En } & \text { este } \\
\text { sentido } & \text { Tacón } \\
(1987 & \text { b) } \\
\text { menciona } & \text { que } \\
\text { estos requieren } \\
45 & \% \text { de }\end{array}$ & \multicolumn{3}{|c|}{$\begin{array}{l}\text { Tabla 6. Análisis químico } \\
\text { proximal de biomasa de } \\
\text { Artemia franciscana cepa } \\
\text { Virrilá, Perú, (\% de peso seco) } \\
\text { obtenida con harina de soya y } \\
\text { pescado. }\end{array}$} \\
\hline $\begin{array}{l}\text { proteínas en la } \\
\text { dieta, durante la } \\
\text { maduración. En } \\
\text { relación a la }\end{array}$ & Componente & $\begin{array}{l}\text { Harina } \\
\text { de } \\
\text { soya } \\
\text { (HS) }\end{array}$ & $\begin{array}{c}\text { Harina } \\
\text { de } \\
\text { pescado } \\
(\mathrm{HP}) \\
\end{array}$ \\
\hline utilización & Proteína & 53.38 & 65.75 \\
\hline Artemia & Grasa & 16.44 & 9.41 \\
\hline estudios & Fibra & - & - \\
\hline $\begin{array}{l}\text { realizados en } \\
\text { Ecuador y USA }\end{array}$ & Ceniza & 16.65 & 15.03 \\
\hline han mostrado & ELN & 13.53 & 9.81 \\
\hline
\end{tabular}

fresco (poliquetos) utilizado en la maduración de Litopenaeus vannamei ha sido exitosamente reemplazado por biomasa congelada de este microcrustáceo (Dhont \& Van Stappen, 2003).

\section{Conclusiones.}

De los resultados obtenidos concluimos que es posible producir biomasa de Artemia franciscana, cepa Perú, con insumos disponibles en el mercado local y que HS proporciona buenos rendimientos en biomasa, la cual también puede ser utilizada junto con otros insumos, en dietas para alimentación de peces y crustáceos.

\section{Agradecimientos.}

El presente trabajo se realizó con financiamiento del Instituto del Mar del Perú (IMARPE), en el marco del proyecto "Reproducción y acondicionamiento del lenguado Paralichthys adspersus / producción de alimento vivo". Se agradece el asesoramiento del Dr. Rafael Tizol del Centro de Investigaciones Pesqueras de la Habana, Cuba, por sus valiosas sugerencias y revisión del manuscrito. A

la Ing. Lili Carrera por su colaboración durante la ejecución del trabajo.

\section{Literatura citada.}

Bransdena M.P., Battaglenea S.C., Moreheada D.T., Dunstanb G.A. \& Nichols P.D. 2005. Effect of dietary 22:6n-3 on growth, survival and tissue fatty acid profile of striped trumpeter (Latris lineata) larvae fed enriched Artemia. Aquaculture. 243: 331-344.

Cochran W. \& Cox G.M. 1997. Diseños experimentales $3^{\text {a }}$ ed. México. Editorial Trillas.

De la Higuera M. \& Cardenete G. 1987. Tecnología de fabricación de piensos para la acuicultura. Alimentación en Acuicultura (FEUGA). España. : 59129.

Devresse B. 1999. Formulación de alimentos para camarón: Las deficiencias nutricionales más frecuentes en alimentos para Litopenaeus vannamei. Panorama Acuícola.: 8-11.

Dhont J. \& Van Stappen G. 2003. Biology, tank production and nutritional value of Artemia. En : Stottrup J.G. \& McEvoy L.A. (Eds.). Live Feeds in Marine Aquaculture. Blackwell Science. : 65-121.

Food and Agriculture Organizatio (F.A.O). 1983. Fish feeds and feeding in developing countries. An interim Report on the ADCP Feed Development Programe. Aquaculture Development and Coordination Programme. Roma.

Fraga I., Galindo J., Reyes R., Alvarez S., Gallardo N., Forrellat A. \& Gonzalez R. 1996. Evaluación de diferentes fuentes proteicas para la alimentación del camarón blanco Penaeus schmitti. Revista Cubana de Investigaciones Pesqueras. 20(1): 6-9.

Gallardo N., González R., Carrillo O., Valdez O. \& Forrellat A. 1989. Una aproximación a los requerimientos de aminoácidos esenciales de Penaeus schmitti. Revista de Investigaciones Marinas.10(3): 259-267.

Godínez D.E., Gallo M.C., Gelabert R., Díaz A.H., Gamboa J., Landa V. \& Godínez E.M. 2004. Crecimiento larvario de Artemia franciscana (Kellog 1906) alimentada con dos especies de microalgas vivas. 
Zootecnia Tropical. 22(3): 265-275.

Haga Y., Tarui F., Ohta K., Shima Y. \& Takeuchi T. 2006. Effect of light irradiation on dynamics of vitamin A compounds in rotifers and Artemia. Fisheries Science. 72: 1020-1026.

Hoff F. \& Snell T. 1993. Plankton culture manual. Third edition . Florida Aquafarms, Inc.: 91-109 .

Lavens P. \& Sorgeloos P. (Eds.). 1996. Manual on the reproduction and use of live food for aquaculture. FAO Fisheries Technical paper. No 361 . Roma, FAO.

Monroig O., Navarro J.C., Amat F., González P. \& Hontoria F. 2006. Effects of nauplial density, product concentration and product dosage on the survival of the nauplii and EFA incorporation during Artemia enrichment with liposomes. Aquaculture. 261: 659-669.

Monroig O., Navarro J.C., Amat F., González P. \& Hontoria F. 2007. Oxidative stability and changes in the particle size of liposomes used in the Artemia enrichment . Aquaculture. 266: 200-210.

Napolitano G. E., Ackman R. \& Ratnayake W. 1990. Fatty acid composition of three cultured algal species (Isochrysis galbana, Chaetoceros gracilis and Chaetoceros calcitrans ) used as food for bivalve larvae. World Aquaculture Society. 21(2): 122-130.

Persoone G., Sorgeloos P., Roels O. \& Jaspers E. 1980. The brine shrimp Artemia . (Eds.) Universa Press. Wetteren, Belgium.

Quinn G.P. \& Keough M.J. 2002. Experimental design and data analysis for biologist. Cambridge University Press.

Robin J.H. 1995. The importance of n-6 fatty acids in the culture of marine fish larvae. ICES mar. Sci. Symp. 201: 106-111.

Sorgeloos P., Lavens P. Leger W. Tackaert \& Versichele D. 1986. Manual for the culture and use of brine shrimp Artemia in aquaculture. State University of Ghent, Belgium.

Seoka M., Kurata M. \& Kumai H. 2007. Effect of docosahexaenoic acid enrichment in Artemia on growth of Pacific bluefin tuna Thunnus orientalis larvae. Aquaculture.270: 193-199.Ba

Steel G.D. \& Torrie J.H. 1995. Bioestadística : Principios y Procedimientos, 2da. ed. MacGraw Hill/Interamericana de México.

Stottrup J.G. \& McEvoy L.A. (Eds.). 2003. Live Feeds in Marine Aquaculture. Blackwell Science. UK.

Suprayudi A., Takeuchi T. \& Hamasaki K. 2004. Effects of Artemia enriched with eicosapentaenoic and docosahexaenoic acid on survival and occurrence of molting failure in megalop larvae of the mud crab Scylla serrata Fisheries Science.70: 650-658.

Tacon A. 1987 (a). The nutrition and feeding of farmed fish and shrimp. A training manual. 1. The essential nutrients. Field document. S/E FAO.

Tacon A. 1987 (b). The nutrition and feeding of farmed fish and shrimp. A training manual. 2. Nutrient sources and composition. Field document. S/E FAO.

Tizol R. 1994. Uso de la levadura torula (Torulopsis utilis) en la obtención de biomasa de Artemia. An. Inst. Invest. Mar. Punta Betín. 23: 165-171.

Torrentera L. \& Tacon A. 1989. La producción de alimento vivo y su importancia en acuacultura. Documento de campo No 12. Proyecto Aquila, FAO.

Van Stappen G. 1996. Introduction, biology and ecology of Artemia. En: P. Lavens \& P. Soorgeloos (Eds). Manual on the production and use of live food for aquaculture. FAO Fisheries technical paper. : 79-251.

Versichele D., Leger P., Lavens P. \& Sorgeloos P. 1991. El uso de Artemia. Acuicultura, Gilbert Barnabé. Ediciones Omega, S.A. Barcelona. : 200-215.

Verschuere L., Rombaut G., Huys G., Dhont J., Zorruelos P., \& Verstraete W. 1999. Microbial control of the cultura of Artemia juveniles through preemptive colonization by selected bacterial strains. Appl Environ Microbiol. 65(6): 2727-2533.

Vinatea J.E., Olivera A. \& Vinatea L. 1993. Producao de biomassa de Artemia em tanques de concreto com fertilizantes inorgánicos e orgánicos na Barra da Lagoa. Universidad Federal de Santa Catarina, Florianópolis, Brasil.

Vinatea L. 1995. Biología, cultivo y uso en Acuicultura del Camarón de Salmuera, Artemia sp. Universidad Nacional Mayor de San Marcos, Escuela de PostGrado.

Vinatea L. 1999. Manual de producción de Artemia (quistes y biomasa) en módulos de cultivo. Universidad Autónoma Metropolitana, México, Unidad Xochimilco, División de Ciencias Biológicas y de la Salud.

Vinatea E., Vega A. \& Adrianzén E. 2002. Artemia Camarón de Salmuera : Bioecología y Nutrición. Tomo I. Dirección de Post Grado de la Facultad de Oceanografía, Pesquería y Ciencias Alimentarias de la U.N.F.V. Lima, Perú.

Xunta de Galicia. 1991. Guía metodológica para la elaboración de estudios de impacto ambiental. Cultivos Marinos. Concellería de ordenación do territorio e obras públicas. Dirección Xeral de Calidade Medioambiental e Urbanismo. España.

Zelaya O., Davis A. \& Rouse D.B. 2007. The influence of Artemia and algal supplements during the nursery phase of rearing pacific white shrimp, Litopenaeus vannamei. journal of the world aquaculture society. 38(4): 486-496.

Zimmermann S., Magalhaes E., De Souza S. \& Penz A. 1989. Effects of three different protein sources in growout feeds for fresh water prawns Macrobrachium rosembergii (de Man) reared in nursery. En: III Simposio Brasileiro sobre Cultivo de Camarao. Joao Pessoa, Paraiba, Brasil (15 a 20 octubre de 1989). II: 257-287.

1 Unidad de Investigaciones en Acuicultura, Instituto del Mar del Perú, Ap. 22, Chucuito, Callao, rcisneros@imarpe.gob.pe

2 Profesor invitado Maestría en Acuicultura, Facultad de Ciencias Biológicas, UNMSM, Virrey José Abascal 293, San Isidro, Lima, aditavega@yahoo.com 